Science, Technology and Development 35 (1): 16-21, 2016

ISSN 0254-6418 / DOI: 10.3923/std.2016.16.21

(C) 2016 Pakistan Council for Science and Technology

\title{
Characterization of PVK Polymeric Material Using Rotating Polarizer and Analyzer Ellipsometer with a Speed Ratio 1:1
}

\author{
Anas A. Alkanoo, Taher M. El-Agez, Sofyan A. Taya and Ahed Afghjani \\ Department of Physics, Islamic University of Gaza, Gaza, Palestinian Authority
}

\begin{abstract}
A homemade spectroscopic ellipsometer is designed indigenously to characterize $\mathrm{Si}_{-} \mathrm{SiO}_{2}$ sample and two Poly (9-vinylcarbazole) (PVK) films. In the constructed ellipsometer, the polarizer and analyzer rotate in the same direction with the same angular speed. The ellipsometric parameters $\psi$ and $\Delta$ are measured as a function of the wavelength for the three samples. The TF Companion software for thin film analysis is used to analyze the experimental results and obtain the optical parameters and thickness of these samples. The dispersion of the refractive index of $\mathrm{SiO}_{2}$ and PVK thin films is found to obey Cauchy's equation. The results reveal an extremely accurate device for thin film characterization.
\end{abstract}

Key words: Ellipsometry, PVK, Refractive index, cauchy’s dispersion

\section{INTRODUCTION}

Carbazole is an aromatic tricyclic compound, which was discovered in 1872. When a large number of carbazole monomers are linked together, poly (vinylcarbazole) compound is formed. Pure carbazole is a white crystalline organic material, which has melting point of $246^{\circ} \mathrm{C}$ and $167.2 \mathrm{~g} /$ mole molecular weight. Carbazole has high boiling point compared to many organic materials. It is an electroluminescent material and emits strong fluorescence and long phosphorescence when excited with UV light. Poly (9-vinylcarbazole) (PVK) is a tricyclic organic polymeric material which is transparent plastic material. The softening point of PVK is nearly $175^{\circ} \mathrm{C}$ and glass transition temperature is $211^{\circ} \mathrm{C}$. The solubility of PVK is very strong in alcohol, ether and tetrahydrofuran (THF) and PVK films are usually deposited by spin coating. It has good optical properties and strong photoconductive and electroluminescence properties. Electrically, PVK is an insulator in dark but under the ultraviolet radiation it exhibits electroconductivity. Commercially PVK can be used in the fabrication of Organic Light Emitting Diodes (OLEDs), sensors and transistors. In OLEDs, PVK is used as a hole transport material. The chemical structures of Carbazole, $\mathrm{N}$-vinylcarbazole and Poly (N-vinylcarbazole) are shown in Fig. 1a-c, respectively.

Light polarization is considered a rich source of information and its measurement is of high significance in many applications. In ellipsometry, the change in the state of polarization of incident light can be measured. If linearly polarized light of known orientation is reflected from a surface, the reflected light will be elliptically polarized. Spectroscopic ellipsometry is of high importance to wide research areas from semiconductors to organic materials. An ellipsometer measures two angles: namely $\Psi$ and $\Delta$. These represent the amplitude ratio $\Psi$ and phase difference $\Delta$ between light waves known as p- and s-polarized light waves. In ellipsometry, an incidence angle is usually chosen so that the sensitivity for the measurement is maximized. For semiconductor characterization, the incidence angle is usually taken in the range $70^{\circ}-80^{\circ}$. Spectroscopic ellipsometry has been efficiently applied in thin film characterization. Moreover, it has been used in the diagnosis on the atomic scale from real-time observation.

Recently, many ellipsometric configurations have been proposed. In 1973, rotating analyzer ellipsometer
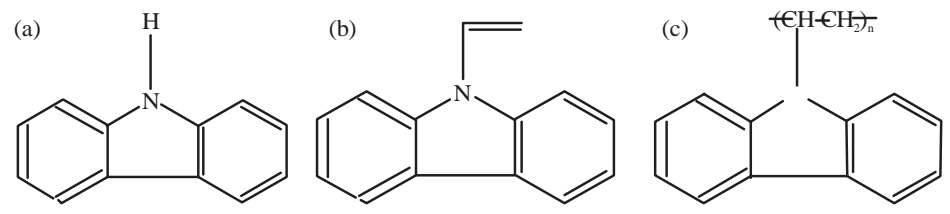

Fig. 1(a-c): Structure of (a) Carbazole, (b) N-vinylcarbazole, (c) and Poly (N-vinylcarbazole) 
Sci. Technol. Dev., 35 (1): 16-21, 2016

was constructed (Aspnes, 1973). Rotating polarizer and analyzer ellipsometer was also proposed (Huang and Chu, 2000; Taya and El-Agez, 2011a) in different speed ratios (El-Agez et al., 2010, 2011; El-Agez and Taya, 2010, 2011, 2012, 2014; Vedam, 1998; Chen et al., 1994; Taya and El-Agez, 2011b, 2012, 2013; An et al., 2002; Azzam, 1978; Taya et al., 2011, 2012). Different ellipsometric configurations involving fixed and rotating phase retarder were also presented and constructed (Opsal et al., 1998; Lee et al., 1998; Taya et al., 2013, 2014; Alkanoo et al., 2015).

In this study, a homemade spectroscopic ellipsometer is used to characterize $\mathrm{Si}_{-} \mathrm{SiO}_{2}$ sample and two PVK films. The thickness and optical parameters of the films are measured in the spectral range $300-800 \mathrm{~nm}$. In the constructed ellipsometer, the polarizer and analyzer rotate with angular speed $\omega$ in the same direction. Commercial software for thin film analysis (TF Companion-optical metrology software) purchased from Semiconsoft, Inc is used to analyze the experimental results.

\section{MATERIALS AND METHODS}

The theory of the constructed ellipsometer is explained in details in refs. (El-Agez et al., 2010; El-Agez and Taya, 2010, 2014). For the sake of clarity, we mention here part of this theory. A schematic diagram of the constructed ellipsometer is shown in Fig. 2. It consists of a well-collimated beam of monochromatic unpolarized light, a fixed polarizer, rotating polarizer with angular speed $\omega$, sample to be characterized, rotating analyzer with the same angular speed as that of the polarizer and detector. The detected intensity can be calculated using Mueller formalism (Taya et al., 2012, 2013).

The Fourier transform of the detected signal can be written as:

$$
I(t)=\alpha_{0}+\alpha_{1} \cos 2 \omega t+\alpha_{2} \cos 4 \omega t+\alpha_{3} \cos 6 \omega t
$$

where, the coefficients $\mathrm{a}_{0}, \mathrm{a}_{1}, \mathrm{a}_{2}$ and $\mathrm{a}_{3}$ are given by:

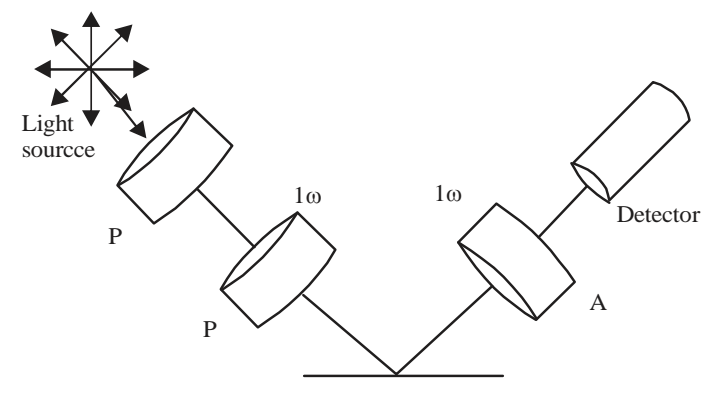

Fig. 2: Schematic diagram of the constructed ellipsometer

$$
\begin{gathered}
\mathrm{a}_{0}=\frac{3}{2}-\cos (2 \psi)+\frac{1}{2} \sin (2 \psi) \cos (\Delta-\delta) \\
\mathrm{a}_{1}=\frac{7}{4}-2 \cos (2 \psi)+\frac{1}{4} \sin (2 \psi) \cos (\Delta) \\
\mathrm{a}_{2}=\frac{1}{2}-\cos (2 \psi)-\frac{1}{2} \sin (2 \psi) \cos (\Delta) \\
\mathrm{a}_{3}=\frac{1}{4}[1-\sin (2 \psi) \cos (\Delta)]
\end{gathered}
$$

The ellipsometric parameters $\psi$ and $\Delta$ can be determined, using the AC Fourier coefficients as follows:

$$
\begin{gathered}
\tan \psi=\frac{\sqrt{\mathrm{a}_{1}+\mathrm{a}_{3}}}{\sqrt{\mathrm{a}_{1}-4 \mathrm{a}_{2}+9 \mathrm{a}_{3}}} \\
\cos \Delta=\frac{-3 \mathrm{a}_{3}+\mathrm{a}_{1}-2 \mathrm{a}_{2}}{\sqrt{\left(\mathrm{a}_{1}+\mathrm{a}_{3}\right)\left(\mathrm{a}_{1}-4 \mathrm{a}_{2}+9 \mathrm{a}_{3}\right)}}
\end{gathered}
$$

Figure 2 shows the main components of the constructed ellipsometer. It consists of (1) 150 watt Super-Quiet Xe arc lamp (Hamamatsu L2274), (2) A fixed polarizer (KARL LAMBRECHT, MGTYE5, Glan-Taylor Ultra-Violet Prism), (3) A rotating polarizer identical to that described in item 2 . This polarizer can be rotated using a stepper motor, (4) The sample to be characterized, (5) A rotating analyzer identical to that described in item 2 and (6) A silicon photodiode (Hamamatsu S1226-8BQ). Michelson interferometer was employed with the sample being one of the arms for the precise sample alignment.

After detection of the signal by the silicon photodiode, it is amplified by a photodiode transimpedance amplifier using LMP7721 operational amplifier. The amplified photosignal is recorded by NI USB 6251 16-bit data acquisition card. Moreover, the control of the stepper motors and the monochromator is achieved through data acquisition card making use of LabView program.

The rotating polarizer is adjusted first using the Brewster angle of incidence for p-polarized light. The analyzer is then aligned to the position where intensity extinction is achieved (El-Agez and Taya, 2011, 2014).

The PVK (Sigma-Aldrich, USA) with an average molecular weight of $11 \times 10^{5}$ was used in this work. $30 \mathrm{mg}$ of PVK was weighed by using a sensitive electrical balance (model N3.ESJ182-4) with a resolution of $10^{-4} \mathrm{~g}$ and dissolved in a proper amount of mixed solvents of toluene and THF. Few drops of the solution were placed 
on a cleaned Si wafer. Inclined coating method was used to form the PVK films. Two PVK films of different thicknesses were prepared. The film thickness was varied by changing the volume of the mixed solvents in the solution. Each sample was then mounted in the sample holder and a beam of light is allowed to impinge on the sample at an incidence angle $\theta_{0}=70^{\circ}$.

\section{RESULTS AND DISCUSSION}

Three samples were studied. The first one (S1) is an ellipsometry standard (Ocean Optics R1 Silicon-Silicon Dioxide Thin Film Reference Wafer, USA). S1 sample is a STEP-WAFER ( $\mathrm{Si}_{-} \mathrm{SiO}_{2}$-step-wafer) $100 \mathrm{~mm}$ diameter. The following is the detailed data given in the calibration data sheet for this sample at the wavelength of $632.8 \mathrm{~nm}$ : $\psi=24.5^{\circ}, \Delta=91.3^{\circ}$, thickness $=506.93 \mathrm{~nm}$ and $\mathrm{n}=1.459$. The second (S2) and third (S3) samples are two thin PVK films deposited on Si substrate. Fig. 3-5 show the ellipsometry spectra obtained with the constructed

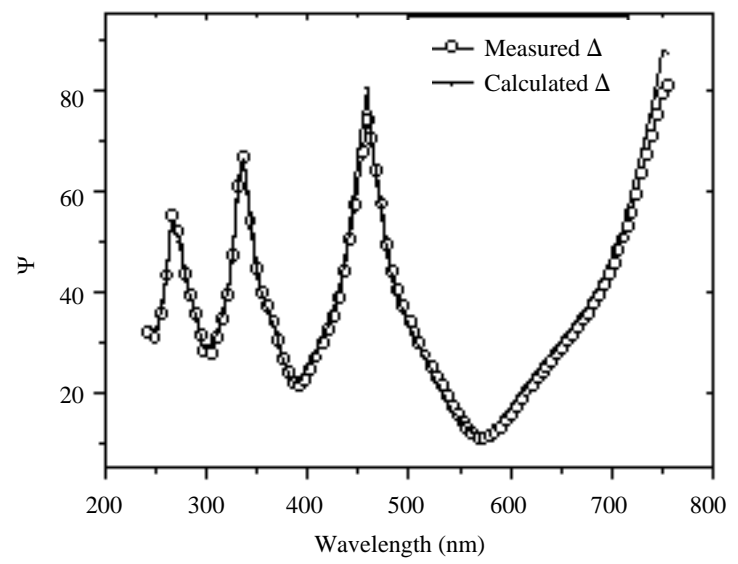

Fig. 3: Psi of $\mathrm{SiO}_{2}$ thin film on silicon wafer as a function of wavelength for ellipsometry S1 sample

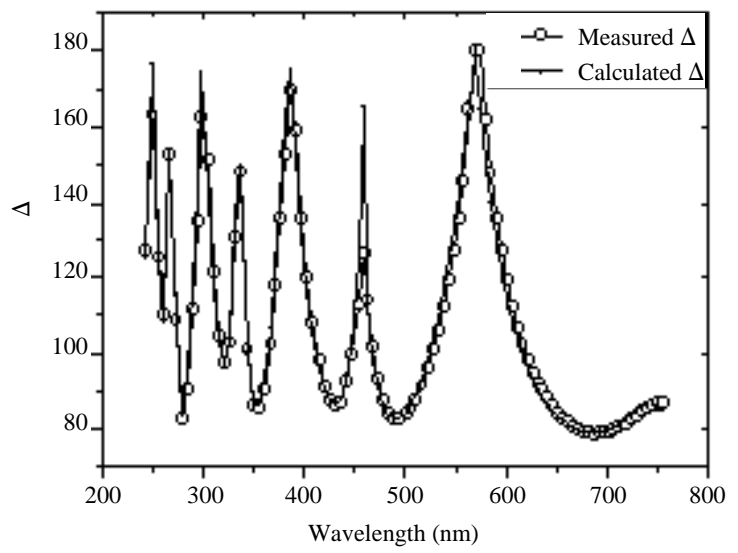

Fig. 4: Delta of $\mathrm{SiO}_{2}$ thin film on silicon wafer as a function of wavelength for ellipsometry S1 sample ellipsometer for S1. Measured and calculated values of $\psi$ and $\Delta$ of S1 versus the wavelength are shown in Fig. 3 and 4, respectively. Experimental and accepted values of $\mathrm{SiO}_{2}$ (S1 sample) are plotted in Fig. 5. Figure 6-8 show the same parameters for S2 sample, whereas, Fig. 9-11 show the same parameters for S3 sample. Discrete Fourier analysis was performed to deduce the normalized Fourier coefficients for each photon energy and then the ellipsometric parameters ( $\psi$ and $\Delta$ ) were obtained using Eq. 6 and 7. The TF Companion software is then used to analyze the results and extract the thickness and the refractive index of the samples. The TF Companion is powerful software for characterization of thin films. The optical measurements are indirect in that they are measuring optical response of the physical properties not the properties themselves (El-Agez and Taya, 2011). One needs to solve an "Inverse problem" in order to find the value of actual physical properties. The "Inverse problem" is solved numerically by finding the best fit

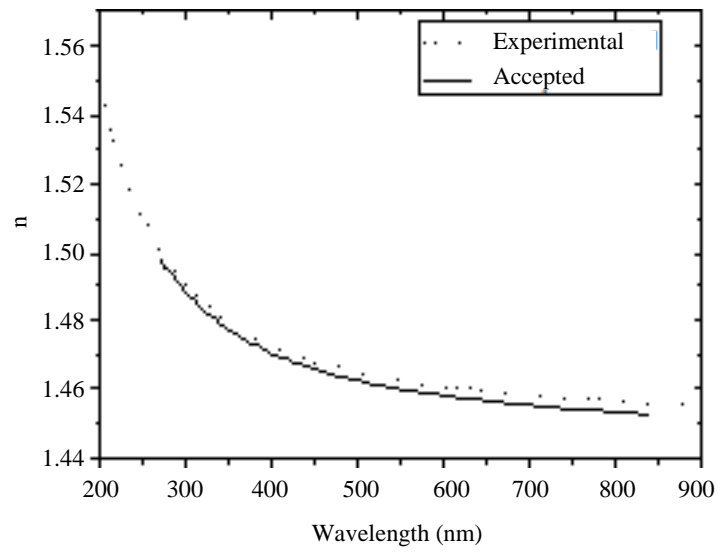

Fig. 5: Refractive index of $\mathrm{SiO}_{2}$ thin film on silicon wafer as a function of wavelength for ellipsometry S1 sample

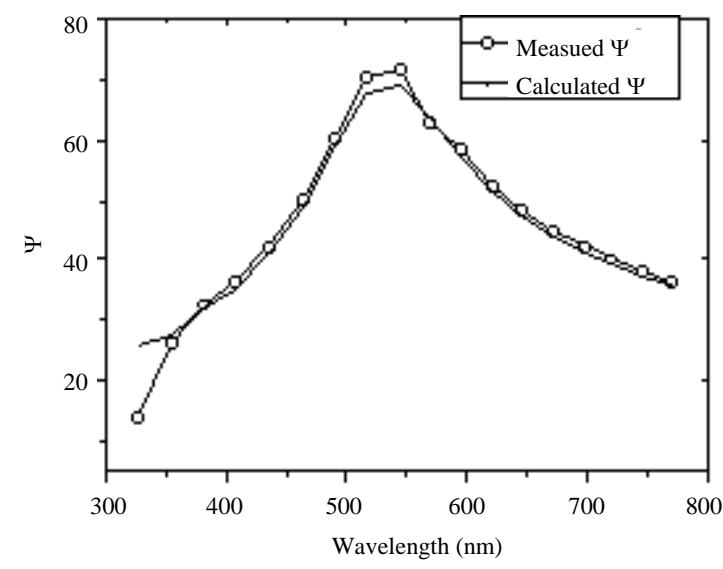

Fig. 6: Psi of PVK thin film on silicon wafer as a function of wavelength for S2 sample 


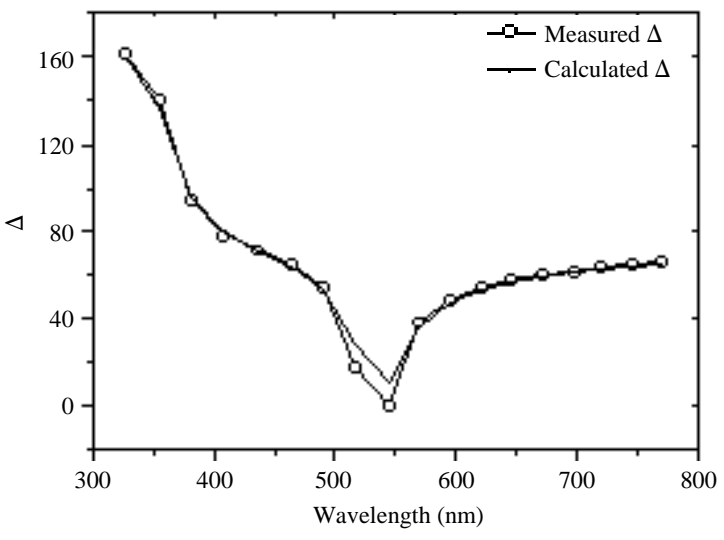

Fig. 7: Delta of PVK thin film on silicon wafer as a function of wavelength for S2 sample

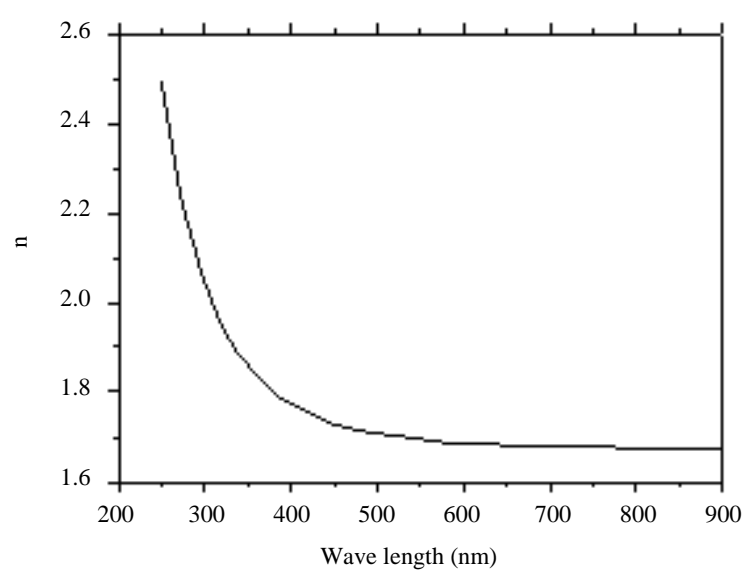

Fig. 8: Refractive index of PVK thin film on silicon wafer as a function of wavelength for S2 sample

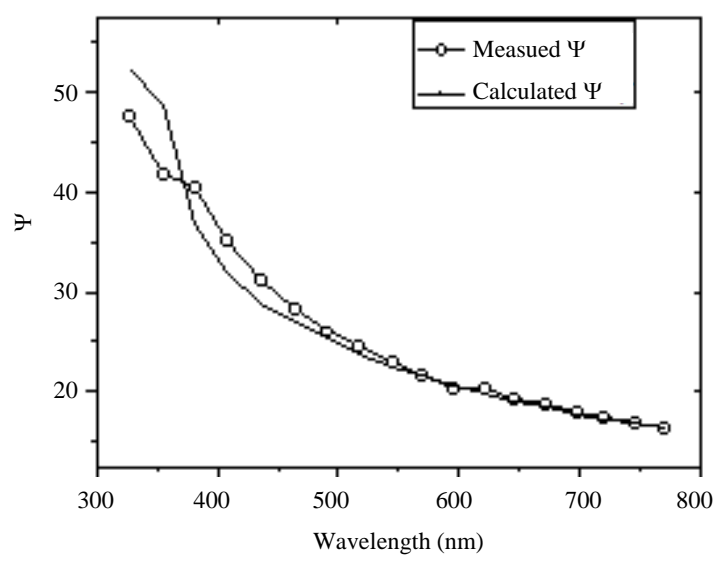

Fig. 9: Psi of PVK thin film on silicon wafer as a function of wavelength for S3 sample

between measured and calculated data. Physical properties are inferred from the model that gives the best

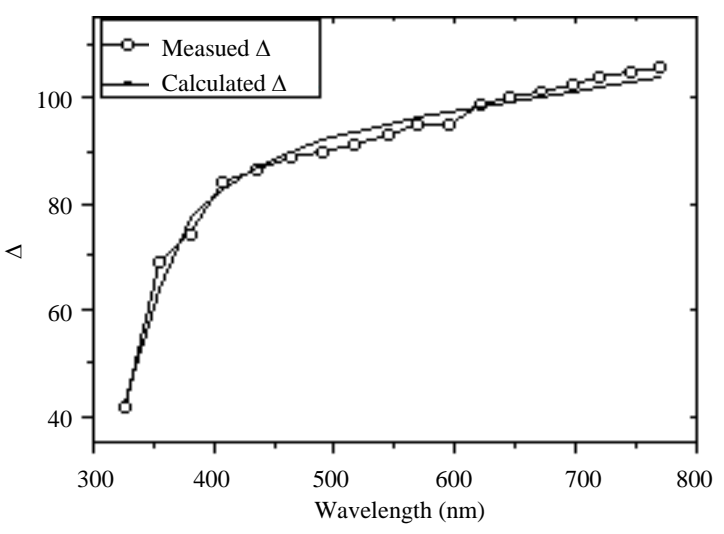

Fig. 10: Delta of PVK thin film on silicon wafer as a function of wavelength for S3 sample

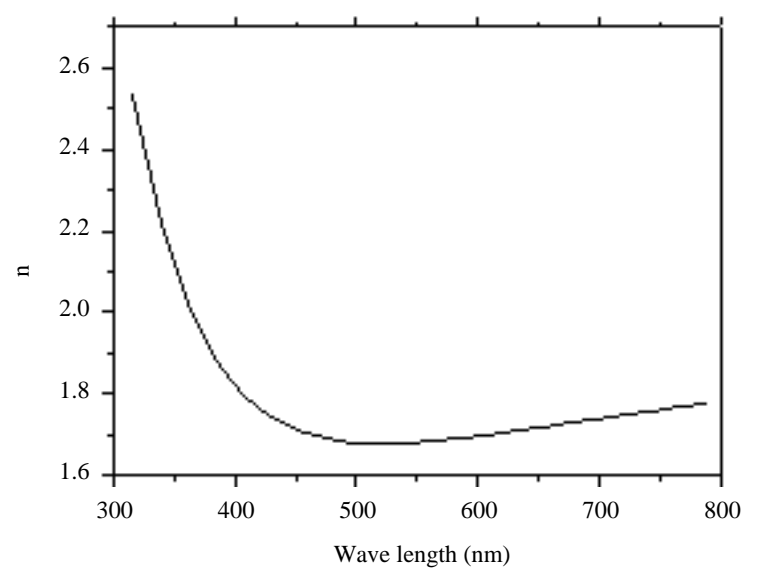

Fig. 11: Refractive index of PVK thin film on silicon wafer as a function of wavelength for S3 sample

fit. General solution is achieved by numerical methods of minimizing the difference between measured (target) data and simulated data, using selected optical model of the filmstack. Selected parameters of the filmstack are adjusted until the minimum error is achieved. The TF Companion uses modified Marquardt-Levenberg minimization that gives fast and robust convergence.

The model adopted for $\mathrm{S} 1$ is ambient- $\mathrm{SiO}_{2}-\mathrm{Si}$ and for $\mathrm{S} 2$ and S3 is ambient-PVK-Si. Since $\mathrm{SiO}_{2}$ and PVK are dielectric materials with a smooth dispersion, Cauchy approximation has been used to represent this dispersion. Solid lines in Fig. 3-11 represent the calculated ellipsometric parameters, using Eq. 6 and 7, whereas, circles represent the experimental data. The measured thickness obtained from TF Companion software was $506.93 \pm 0.43 \mathrm{~nm}$ for S1, $94.48 \pm 0.745 \mathrm{~nm}$ for S2 and $35.33 \pm 0.00068 \mathrm{~nm}$ for S3. Moreover, the spectroscopic measurements of the refractive index of the $\mathrm{SiO}_{2}$ and PVK films in the spectral range $300-800 \mathrm{~nm}$ are shown in Fig. 5, 8 and 11 for S1, S2 and S3, respectively. As can be 
Sci. Technol. Dev., 35 (1): 16-21, 2016

Table 1: Cauchy's constants for the three samples

\begin{tabular}{lccc}
\hline Samples & $\mathrm{N}_{0}$ & $\mathrm{~N}_{2}$ & $\mathrm{~N}_{4}$ \\
\hline $\mathrm{SiO}_{2}$ film on Si wafer $(506.93 \mathrm{~nm})$ & 1.451 & 0.0033 & $5 \times 10^{-5}$ \\
The first sample of PVK film on Si wafer $(94.48 \mathrm{~nm})$ & 1.681 & -0.0081 & 0.00367 \\
The second sample of PVK film on Si wafer $(35.33 \mathrm{~nm})$ & 1.979 & -0.1625 & 0.02196
\end{tabular}

seen from the figures, at $\lambda=632.8 \mathrm{~nm}$, the measured index was 1.459 with percent error of $0.05 \%$ for $S 1,1.683$ with a percent error of $1.5 \%$ for S2 and 1.71 with percent error $0.0013 \%$ for S3.

The following equation describes the behavior of refractive index as a function of wave-length $\lambda$. It is known as Cauchy's equation:

$$
\mathrm{n}(\lambda)=\mathrm{N}_{\mathrm{o}}+\frac{\mathrm{N}_{2}}{\lambda^{2}}+\frac{\mathrm{N}_{4}}{\lambda^{4}}
$$

where, $\mathrm{N}_{0}, \mathrm{~N}_{2}$ and $\mathrm{N}_{4}$ are called Cauchy's constants.

Table 1 shows the values of $\mathrm{N}_{0}, \mathrm{~N}_{2}$ and $\mathrm{N}_{4}$ in Cauchy relation for $\mathrm{SiO}_{2}$ film on $\mathrm{Si}$ wafer and two samples with different thickness of PVK film on Si substrate.

\section{CONCLUSION}

A homemade rotating polarizer and analyzer spectroscopic ellipsometer was used to characterize $\mathrm{Si}_{-} \mathrm{SiO}_{2}$ and two PVK films deposited on $\mathrm{Si}$ wafer. The speed ratio at which the elements rotate was 1:1. The ellipsometric parameters $\psi$ and $\Delta$ were measured as a function of the wavelength in the spectral range 300-800 nm. Commercial software for thin film analysis (TF Companion-optical metrology software) obtained from Semiconsoft, Inc, was used to analyze the experimental results and extract the optical parameters and thickness of these samples. The dispersion of the refractive index of $\mathrm{SiO}_{2}$ and PVK thin films is found to obey Cauchy's equation. The results revealed high accuracy for thin film characterization.

\section{REFERENCES}

Alkanoo, A.A., S.A. Taya and T.M. El-Agez, 2015. Effect of the orientation of the fixed analyzer on the ellipsometric parameters in rotating polarizer and compensator ellipsometer with speed ratio 1: 1. Opt. Quantum Electron., 47: 2039-2053.

An, I., M.G. Park, K.Y. Bang, H.K. Oh and H. Kim, 2002. Development of multichannel ellipsometry with synchronously rotating polarizer and analyzer. Jpn. J. Appl. Phys., 41: 3978-3980.

Aspnes, D.E., 1973. Fourier transform detection system for rotating-analyzer ellipsometers. Opt. Commun., 8: 222-225.
Azzam, R.M.A., 1978. A simple Fourier photopolarimeter with rotating polarizer and analyzer for measuring Jones and Mueller matrices. Opt. Commun., 25: 137-140.

Chen, L.Y., X.W. Feng, Y. Su, H.Z. Ma and Y.H. Qian, 1994. Design of a scanning ellipsometer by synchronous rotation of the polarizer and analyzer. Appl. Opt., 33: 1299-1305.

El Agez, T.M. and S.A. Taya, 2014. Design of a spectroscopic ellipsometer by synchronous rotation of the polarizer and analyzer in opposite directions. Microwave Optical Technol. Lett., 56: 2822-2826.

El-Agez, T.M. and S.A. Taya, 2010. A fourier ellipsometer using rotating polarizer and analyzer at a speed ratio 1 : 1 . J. Sens., 2010. 10.1155/2010/706829

El-Agez, T.M. and S.A. Taya, 2011. Characterization of $\mathrm{SiO}_{2}$ thin film using rotating polarizer analyzer ellipsometer. Int. J. Microwave Opt. Technol., 6: 363-371.

El-Agez, T.M. and S.A. Taya, 2012. Noise effect on thin film characterization using rotating polarizer analyzer ellipsometer. Acta Physica Polonica A, 122: 15-19.

El-Agez, T.M., A.A. El Tayyan and S.A. Taya, 2010. Rotating polarizer-analyzer scanning ellipsometer. Thin Solid Films, 518: 5610-5614.

El-Agez, T.M., D.M. Wieliczka, C.E. Moffitt and S.A. Taya, 2011. Spectroscopic ellipsometry time study of low-temperature plasma-polymerized plain trimethylsilane thin films deposited on silicon. Phys. Scripta, 84.

Huang, Z. and J. Chu, 2000. Optimizing precision of fixed-polarizer, rotating-polarizer, sample and fixedanalyzer spectroscopic ellipsometry. Appl. Opt., 39: 6390-6395.

Lee, J., P.I. Rovira, I. An and R.W. Collins, 1998. Rotating-compensator multichannel ellipsometry: Applications for real time Stokes vector spectroscopy of thin film growth. Rev. Scient. Instrum., 69: 1800-1810.

Opsal, J., J. Fanton, J. Chen, J. Leng and L. Wei et al., 1998. Broadband spectral operation of a rotatingcompensator ellipsometer. Thin Solid Films, 313-314: 58-61.

Taya, S.A. and T.M. El-Agez, 2011a. Comparing optical sensing using slab waveguides and total internal reflection ellipsometry. Turk. J. Phys., 35: 31-36. 
Taya, S.A. and T.M. El-Agez, 2011b. Ellipsometry of anisotropic materials: A new efficient polynomial approach. Opt. Int. J. Light Electron Opt., 122: 666-670.

Taya, S.A. and T.M. El-Agez, 2012. Effect of noise on the optical parameters extracted from different ellipsometric configurations. Physica Scripta, 85, 4. 10.1088/0031-8949/85/04/045706.

Taya, S.A. and T.M. El-Agez, 2013. Rotating polarizer analyzer ellipsometer with a fixed compensator. Optik-Int. J. Light Electron Optics, 124: 3379-3383.

Taya, S.A., T.M. El-Agez and A.A. Alkanoo, 2011. Thin film characterization using rotating polarizer analyzer ellipsometer with a speed ratio 1:3. J. Electromagn. Anal. Appl., 3: 351-358.
Taya, S.A., T.M. El-Agez and A.A. Alkanoo, 2012. Ellipsometric configurations using a phase retarder and a rotating polarizer and analyzer at any speed ratio. Chin. Phys. B, 21. 10.1088/1674-1056/21/11/110701

Taya, S.A., T.M. El-Agez and A.A. Alkanoo, 2013. Rotating polarizer, compensator and analyzer ellipsometry. Chin. Phys. B, 22. 10.1088/16741056/22/12/120703

Taya, S.A., T.M. El-Agez and A.A. Alkanoo, 2014. A spectroscopic ellipsometer using rotating polarizer and analyzer at a speed ratio 1: 1 and a compensator. Optical Quantum Electron., 46: 883-895.

Vedam, K., 1998. Spectroscopic ellipsometry: A historical overview. Thin Solid Films, 313-314: 1-9. 